\title{
RACIONALIZACIÓN DE LOS PROCESOS EN LA REVISIÓN FÍSICA DE INVENTARIOS EN EL ÁREA DE ALMACENAMIENTO DE PRODUCTOS TERMINADOS GARANTIZANDO LA SEGURIDAD Y SALUD EN EL TRABAJO ${ }^{1}$
}

\author{
Rationalization of processes in the physical review of inventories in the \\ storage area of products finished guaranteeing safety and health at job
}

ANGIE ALEJANDRA LINARES BASABE², YESICA LORENA AGUIRRE ARIAS ${ }^{3}$, MANUEL ALFONSO MAYORGA MORATO ${ }^{4}$

Recibido:08 de mayo de 2021. Aceptado:08 de junio de 2021

DOI: http://dx.doi.org/10.21017/rimci.2021.v8.n16.a104

\begin{abstract}
Resumen
La compañía sobre la cual se hizo el presente estudio realiza una vez al mes, de forma técnica, la revisión física del inventario, con un nivel de confianza y exactitud del 100\%, aunque su nivel de precisión es muy alto, es necesario cambiar la forma en cómo se está ejecutando el control de inventarios, puesto que el método actual expone al operario a un riesgo innecesario e infalible, lo que ha motivado al jefe de operaciones a solicitar el cambio del proceso. Sin embargo, el proceso con el que se está verificando el inventario en la actualidad requiere de más horas hombre y el pago de horas extras a sus trabajadores para culminar la actividad, generando un aumento en los costos nominales. Por esta razón, el objetivo del presente trabajo consiste en optimizar el método de operación en la revisión física del inventario, permitiendo mejorar su efectividad, disminuir los costos nominales, tiempos de ejecución y garantizando la seguridad y salud en el trabajo.

Por ende, el trabajo se ejecutó mediante una investigación aplicada descriptiva, desarrollando estrategias que parten de teorías, con el fin de aplicarlas y así darle una solución óptima a la problemática encontrada en la compañía. Así mismo, se implementó una investigación cuantitativa en donde los datos surgen de un trabajo de campo realizado en el área de almacenamiento de productos terminados, por medio de un estudio de tiempos y movimientos en la revisión de inventarios. Adicionalmente, se realizó un análisis de costos, acompañado de diversas simulaciones que permitieron establecer cuál es la maquinaria adecuada para el proceso. Como resultado, se pudo determinar que el dron y el método zigzag es la solución óptima para la revisión física del inventario al disminuir los riesgos, costos y tiempos de ejecución.
\end{abstract}

Palabras clave. Revisión física; inventarios; seguridad y salud en el trabajo; estudio de trabajo; simulación.

\section{Abstract}

The company on which this study was carried out performs a technical review of the inventory once a month, with a level of confidence and accuracy of $100 \%$, although its level of precision is very high, it is necessary to change the how

1 Artículo basado en el proyecto investigativo de la racionalización de la revisión física del inventario en el área de almacenamiento en una compañía distribuidora de productos terminados, ejecutado en el año 2020 y 2021 desde el programa de ingeniería industrial de la Universidad Libre, sede Bogotá

2 Estudiante de ingeniería industrial, Universidad Libre, Colombia. Correo electrónico: angiea-linaresb@unilibre.edu.co

3 Estudiante de ingeniería industrial, Universidad Libre, Colombia. Correo electrónico: yesical-aguirrea@unilibre.edu.co

4 Ingeniero industrial. Universidad INCCA, Colombia. Director del programa Ingeniería de producción por ciclos Universidad Distrital de Bogotá, Colombia. Correo electrónico: mamayorgam@udistrital.edu.co 
inventory control is being executed, since the current method exposes the operator to unnecessary and infallible risk, which has motivated the operations manager to request a change in the process. However, the process with which the inventory is currently being verified requires more man hours and the payment of overtime to its workers to complete the activity, generating an increase in nominal costs. For this reason, the objective of this work is to optimize the method of operation in the physical review of the inventory, allowing it to improve its effectiveness, reduce nominal costs, execution times and guaranteeing safety and health at work.

Therefore, the work was carried out through descriptive applied research, developing strategies that start from theories, in order to apply them and thus give an optimal solution to the problems found in the company. Likewise, a quantitative investigation was implemented where the data emerged from a field work carried out in the finished product storage area, through a study of times and movements in the inventory review. Additionally, a cost analysis was carried out, accompanied by various simulations that made it possible to establish which is the appropriate machinery for the process. As a result, it was determined that the drone and the zigzag method is the optimal solution for the physical review of inventory by reducing risks, costs and execution times.

Keywords. Physical review; inventories; occupational health and safety; work study; simulation.

\section{INTRODUCCIÓN}

L A COMPAÑía realiza una vez al mes de forma técnica la revisión física del inventario con un nivel de confianza y exactitud del 100\%. Aunque su nivel de precisión es muy alto, es necesario cambiar la forma en cómo se está ejecutando este control de inventarios, puesto que el método actual expone al operario a un riesgo innecesario e infalible, por este motivo, el jefe de operaciones solicita cambiarlo. El proceso con el que se está verificando el inventario en la actualidad requiere de más horas hombre y el pago de horas extras a sus trabajadores para culminar la revisión física del inventario causando un aumento en los costos.

Antes de la solicitud del proveedor, el método realizado se ejecutaba desde hace nueve años y medio; el cual era:

- Método Horizontal: El operario se sube a una canasta metálica (elaborada específicamente para este procedimiento y con las medidas adecuadas para que encaje en el montacargas), con el arnés de seguridad puesto y conectado a la línea de vida. El operario encargado de manejar el montacargas procede a suspenderlo en el aire y el operario suspendido en el aire procede a leer el UVA (unidad de almacenamiento) con el lector láser de cada fila. El recorrido inicia siempre de izquierda a derecha y vuelve a empezar de la misma forma en la siguiente fila, así sucesivamente por cada una de las filas de los doce racks de almacenamiento, a su vez, el montacargas se moviliza con el operario hasta terminar el control del inven- tario del área de almacenamiento. Con el método horizontal en el área de almacenamiento el cual está conformada por 12 racks, se requieren ocho horas de trabajo estando la bodega entre el $50 \%$ - $60 \%$ de su capacidad y diez horas con una capacidad de almacenamiento en un rango del $70 \%-100 \%$ en la estantería, lo cual equivale a una jornada laboral y a un pago de horas extras cuando la capacidad es mayor o igual al 70\%.

Después de ser solicitado el cambio del método, la compañía tomó las medidas necesarias para hacer este mismo procedimiento, pero con un método diferente, reduciendo el riesgo del operario y utilizando el montacargas de acuerdo con su función. Los métodos que se manejaron fueron el método vertical, método central - vertical y el método en zigzag, los cuales consisten en:

- Método Vertical: Una vez el operario se encuentre dentro de la canasta metálica con el arnés de seguridad y la línea de vida conectada a la misma, el operario del montacargas procede a suspenderlo en el aire mientras el lee el UVA con el lector láser de cada columna e iniciando siempre de abajo hacia arriba y volviendo a empezar de la misma forma en la siguiente columna y así sucesivamente con cada una de las columnas. Esta vez el montacargas no se moviliza con el operario dentro de la canasta metálica, pues una vez leída el UVA de la primera columna, el operario debe descender del montacargas mientras esta se dirige a la segunda columna y una vez se posicione el montacargas, el operario procede a subirse 
nuevamente a la canasta metálica, conecta su línea de vida a la misma y lee el UVA de la segunda columna y así sucesivamente por los doce racks de almacenamiento. Con el método vertical en el área de almacenamiento (conformado por 12 rack), requiere de aproximadamente once horas de trabajo con una capacidad de almacenamiento en bodega del $60 \%$ en la estantería. Por consecuencia, es necesario el pago de horas extras para cada uno de los trabajadores que maneja, dirige y participa en el control físico del inventario, para dar por terminado el proceso ese mismo día.

- Método Central-Vertical: Es similar al método anterior (método vertical) con la diferencia que el montacargas se sitúa en la mitad de dos columnas facilitándole al operario la función de leer el UVA de dos columnas antes de descender del montacargas y así sucesivamente con todas las columnas de los doce racks ubicados en el área de almacenamiento. El método central-vertical tarda aproximadamente nueve horas con una capacidad de almacenamiento (conformado por 12 rack) en la bodega del $60 \%$ en la estantería. Por consecuencia, es necesario el pago de horas extras.

- Método zigzag: Es similar al método horizontal, pero este, empieza en la primera columna de abajo hacia arriba, y de arriba hacia abajo en la segunda columna. Con este método, se pasaría por alto el tiempo de bajada (arriba hacia abajo) como lo hace el método vertical o el método central vertical. El Método zigzag es el más eficiente de todos los métodos nombrados anteriormente, esto debido a que la lectura del UVA se realiza en el proceso de ascenso y descenso que realiza el montacarguista, mientras que los otros métodos, siempre omiten el escaneo del UVA en el proceso de descenso. Lastimosamente, este método fue descartado por la no adaptación de las planillas del sistema a la lectura del UVA, impidiendo que la ruta de lectura se realice exitosamente. El tiempo estimado del proceso con este método, se encontraba entre seis y siete horas hombre, con una capacidad de almacenamiento (conformado por 12 rack) en la bodega del $50 \%$ al $60 \%$.
Estos procesos son inadecuados, debido al riesgo de la integridad física del operario por tres razones:

1. No cuentan con la maquinaria adecuada para trabajo en alturas, puesto que el montacargas solo puede subir y bajar mercancía de forma vertical, además no se puede movilizar con un peso suspendido en el aire porque tiene un gran riesgo de volcarse.

2. Es incongruente tener un arnés de seguridad si la línea de vida no está conectada a un área segura. Por lo tanto, si la máquina se llega a volcar el operario no tiene como salir ileso de la caída, pues su línea de vida está conectada a la canasta de acero, la cual está suspendida en el aire y sujeta al montacargas por medio de cadenas y candados.

3. En el área de los racks (almacenamiento) no existe un sitio seguro para la línea de vida del operario.

De acuerdo con las razones anteriormente expuestas, el proceso de control de inventarios no solo carece de efectividad, también carece de seguridad para los operarios en todo el proceso.

De acuerdo con la explicación se deben tener en cuenta estos factores:

- Aumento de los tiempos en la revisión física del inventario.

El tiempo que incurre un operario en la revisión física del inventario por los métodos mencionados, ocasiona el aumento de los costos y tiempos de ejecución.

- Trabajo en alturas

La altura en la que trabaja el operario es de aproximadamente 11,2 metros, este operario cuenta con los EPP (elementos de protección personal) necesarios para la realización de esta actividad, pero este proceso se realiza con una máquina no apta para trabajos en altura.

- Manejo adecuado del montacargas

El montacargas por sí solo no se puede utilizar para el trabajo en alturas, pero con la 
adecuación de una canastilla o cesta metálica diseñada y certificada para el montacargas con los estándares exigidos por la Resolución 1409 del Ministerio de Protección Social y con las normas ANSI, si es permitido su uso.

\section{II.JUSTIFICACIÓN}

La empresa distribuidora de productos terminados cuenta con una confiabilidad de inventarios del $100 \%$. Para poder mantener este nivel de confiabilidad, la compañía dispone de un costo fijo de contratación, por lo que las horas extras representan un gran valor en los gastos operacionales. Por esto, para poder efectuar una revisión física del inventario y mantener el porcentaje de confiabilidad en la compañía, se debe generar un aumento en la demanda laboral, con el fin de buscar soluciones estratégicas y generar un control en los gastos operacionales.

Con el propósito de disminuir la problemática mencionada anteriormente, se propuso el desarrollo de esta investigación, puesto que, la empresa no cuenta con procedimientos adecuados para llevar a cabo la revisión de inventarios y métodos seguros para el operario, ocasionando un aumento en el tiempo y en el uso de la mano de obra fuera de su jornada laboral.

Por lo tanto, es importante crear un procedimiento óptimo, eficiente, confiable y seguro, que disminuya considerablemente las horas hombre y genere menos riesgo para el trabajador, aportando una solución que no aumente los costos, ni genere demoras en el proceso. Esto con el fin de beneficiar a los operarios con actividades seguras; al jefe de operaciones con la disminución de tiempos en la revisión física del inventario y a la empresa con no incurrir en altos costos nominales.

Además, la compañía ha consultado nuevas formas de realizar el proceso de revisión de inventarios, pero estas soluciones son de alto costo. Por esto, se espera que con la realización del proyecto se encuentre la solución más apropiada para reducir el riesgo del operario y el tiempo, sin aumentar los costos.

\section{MARCo TeÓRICO}

Para comprender las teorías y temas a tratar en la realización y solución del problema presentado en la compañía se tiene en cuenta las siguientes definiciones:

\section{A. Estudio del trabajo}

De acuerdo con Kanawaty el estudio de trabajo es "el examen sistemático de los métodos para realizar actividades con el fin de mejorar la utilización eficaz de los recursos y de establecer normas de rendimiento con respecto a las actividades que se están realizando" [1], teniendo en cuenta la definición anterior se entiende por estudio de trabajo una herramienta de medición que implica determinar el tiempo estándar que tarda la realización de una tarea, considerando los factores que influyen como tiempo ocioso, retrasos y demoras; además se debe tener en cuenta los movimientos que realiza el operario, esto con el fin de reducir los movimientos innecesarios, costos y tiempos.

El estudio de trabajo es la vinculación entre el estudio de tiempos y métodos, esto ayuda a determinar los movimientos que se realizan en una tarea y el tiempo que esto conlleva, para así conseguir simplificar el tiempo y los movimientos innecesarios, para así generar mayor productividad.

El objetivo del estudio de trabajo en esta investigación consiste en tomar los tiempos y movimientos de los métodos anteriormente mencionados en el artículo, con el fin de establecer el método mas eficiente para el proceso.

Para la realización del estudio de trabajo se debe seguir el procedimiento estipulado, el cual cuenta con ocho pasos, que son:

1. Seleccionar el proceso que se va a evaluar

2. Registrar los datos importantes del proceso a evaluar.

3. Examinar e identificar cada uno de los detalles del proceso, determinando la importancia de este, la maquinaria utilizada, los actores que influyen en el proceso y los movimientos innecesarios del proceso

Rev. Ingeniería, Matemáticas y Ciencias de la Información Vol. 8 / Núm. 16 / julio - diciembre de 2021; pág. 77-87 
4. Establecer cuál es el método más apropiado y económico utilizando diferentes técnicas, en este caso se utilizaría la simulación y una investigación de la innovación tecnológica

5. Evaluar los resultados obtenidos comparando la cantidad de trabajo necesario con el nuevo método

6. Definir el nuevo método por medio del estudio realizado, dándolo a conocer a las partes interesadas

7. Reemplazar el método actual por el método planteado.

8. Controlar y vigilar el nuevo método para evaluar que se cumpla con los objetivos expuestos [1].

De los pasos nombrados anteriormente, es importante aclarar que solamente se trabajó hasta el punto 6, puesto que el punto 7 y 8 los decide la compañía de manera optativa.

\section{B. Trabajo en alturas}

Se considera trabajo en alturas a toda actividad que realiza un trabajador a una altura igual o mayor de 1.50 metros, se considera como una actividad de alto riesgo por la alta probabilidad de accidentabilidad y muerte en el lugar de trabajo [2].

Se deben determinar las medidas necesarias y determinar todos los posibles riesgos que puedan existir en los operarios que realizan trabajo en alturas, esto se hace de acuerdo con la Resolución 1409 del 2012.

La Resolución 1409 del 2012 se encarga de establecer un reglamento de seguridad para la protección del empleado contra la caída en el trabajo en alturas [3] que aplica a todos los operarios que realizan la revisión física del inventario, se deben identificar las condiciones inseguras en las alturas, como lo pueden ser: trabajo en máquinas en movimiento, obstáculos, entre otros (...), por ende, se deberán establecer acciones de prevención o correctivas para los accidentes que pueden ocurrir en el trabajo.

Para la protección de los operarios en el trabajo en alturas se debe utilizar distintos elementos de protección personal como: el arnés de cuerpo com- pleto el cual debe ser certificados y deben incluir elementos para conectarse al anclaje, el anclaje puede ser fijo o móvil de acuerdo con la actividad que realizan, pero se recomienda que no esté conectado con la maquinaria en movimiento, puesto que no es un lugar seguro para el operario en caso de accidente. Se debe aclarar que todo el equipo como el arnés, gancho, el anclaje, el conector deben estar certificados como elementos de protección personal seguros, al igual que la competencia laboral y la capacitación para trabajo seguro en alturas para los operarios.

Se deben solicitar pruebas que garanticen el buen funcionamiento del sistema de protección contra caídas, cumpliendo con los estándares y funcionando como acciones preventivas, dentro de estas acciones para el trabajo en alturas se debe contar con un permiso en el trabajo en alturas, y las distintas certificaciones de los operarios y los elementos de protección personal.

En la realización del proyecto se espera tomar medidas en la fuente, utilizando sistemas de ingeniería para la prevención de caídas, intentando buscar una alternativa que disminuya el riesgo del trabajo en alturas o en el mejor de los casos evite el trabajo en alturas para el operario, en caso de realizar este sistema debe estar documentado dentro del programa de protección contra caídas del SG-SST [3].

\section{Innovación tecnológica}

Se entiende como la modificación o la mejora de los bienes, servicios o procesos por medio de tecnología. El objetivo de la innovación tecnológica consiste en determinar una maquinaria mejorada que permita la disminución del tiempo en la revisión física del inventario.

La innovación tecnológica dentro del proyecto va dirigido al tipo incremental que consiste en mejorar los procesos, este tipo no se basa en crear otras tecnologías sino en ofrecer mejoras [4]. El objetivo de la innovación tecnológica en la realización del proyecto es realizar una investigación de las maquinarias en el mercado utilizadas en procesos similares.

\section{Simulación}

La simulación es una herramienta que, apoyada por datos y software, permite evaluar el impacto que 
genera la modificación de variables en un proceso determinado, además, selecciona los recursos o método óptimo para el proceso que se desee analizar.

SIMIO es un software que modeliza, simula y visualiza el comportamiento de un sistema donde existan flujos de materiales, personas o información, tales como: Fabricas que diseñen nuevos layout (disposición de los elementos dentro del alancen), hospitales donde deben gestionar las prioridades de los recursos disponibles, centros de almacenaje, aeropuertos para optimizar una capacitada y su nivel de servicio, etc.

El objetivo de realizar una simulación del sistema con SIMIO es validar los métodos propuestos y seleccionar el óptimo para el proceso de revisión física del inventario [5].

\section{Marco concePtuAL}

Se presentan unas descripciones de los conceptos que sirven para crear una contextualización de las diferentes tareas que se desglosan de la revisión física del inventario y el sistema de gestión de seguridad y salud en el trabajo.

Accidente de trabajo: Se entiende como accidente de trabajo a todo daño físico por causa $u$ ocasión de trabajo.

Anclaje: Es el punto donde el conector une al arnés de seguridad con el anclaje, este puede ser móvil o fijo de acuerdo con la necesidad de la empresa [4].

Arnés de seguridad: Es un elemento de protección personal para el trabajo en alturas .

Conector: Es el elemento que permite unir el arnés de seguridad con el anclaje.

Control de inventarios: El seguimiento que se le hace a los productos, determinar las políticas para realizar este tipo de controles y tomar medidas precisas en relación con el número óptimo de productos que deben mantenerse lo mismo al tamaño de pedidos [6].

Gancho: Es un elemento del conector y permite la unión entre el arnés de seguridad y el conector.
Rack: Estantería ubicada en el área de almacenamiento de productos terminados

Nivel: Es la cantidad de filas que hay en una estantería.

Racionalización: Se puede entender como la racionalización del proceso como aquel que se encarga de la optimización de tiempo, costos y esfuerzo, partiendo de una planificación e intentando reducir los factores anteriormente mencionados [7].

Riesgo: Según la Norma NTC ISO 31000 del 2018 define riesgo como "el efecto de la incertidumbre sobre los objetivos" [8], está caracterizado por la referencia a los eventos potenciales y las consecuencias.

Seguridad y salud en el trabajo: Se encarga de la prevención de accidentes de trabajo y enfermedades laborales, por medio de la determinación de factores que pueden afectar al trabajador y los controles necesarios para evitarlo, esto se realiza de acuerdo con la normativa vigente.

Tiempo global: Hace referencia a la suma de los tiempos totales de cada uno de los racks en el proceso de la revisión de inventarios.

Tiempo de total: Es el tiempo que requiere el trabajador para completar el proceso de revisión de inventarios en un rack.

UVA: Es la unidad de almacenamiento, entendida como la identificación de cada familia de productos.

\section{Metodología}

Este estudio es una investigación aplicada descriptiva, que, desarrollando estrategias las cuales parten de teorías, para aplicarlas y así darle solución a la problemática encontrada, con el fin de analizar y entender la situación actual de la empresa y de esta forma, acercarse a una solución. Así mismo, se implementó una investigación cuantitativa, en donde los datos surgieron de un trabajo de campo realizado en el área de almacenamiento de la compañía, por medio de un estudio 
de tiempos y movimientos en la revisión física del inventario.

Además, la selección de estos dos tipos de investigación se generó debido a la maleabilidad de los datos, la facilidad del enfoque ante una situación, población o fenómeno que surge en el estudio y el análisis profundo que se puede divisar de la información, permitiendo analizar los datos obtenidos en el desarrollo del estudio de tiempos de almacenamiento, el análisis de la productividad y la observación de factores que ralentiza el desarrollo de la revisión del inventario.

Por otra parte, el estudio del trabajo consiste en tomar el tiempo que los operarios se demoran en cada una de las filas que contiene cada rack (estantería) de la bodega; el total del tiempo va a dar como resultado el ciclo del proceso. Sin embargo, el estudio se realizó varias veces para poder definir un ciclo estándar, porque el tiempo estándar, va a ser el periodo de tiempo a disminuir. Del mismo modo, en la toma de tiempos hay varios factores que se tuvieron en cuenta, tales como: la experiencia de los operarios, la resistencia física, la posición de la UVA (código de barras del producto) en la estiba, las condiciones mecánicas del montacargas, la seguridad del operario y los incidentes[9].

Así mismo, una de las herramientas a utilizar luego de recolectar los datos en el estudio de trabajo es la identificación de la maquinaria utilizada en procesos similares de revisión física del inventario, una vez se seleccionó la maquinaria pertinente al proceso, se analizaron los costos de adquisición. Lo anterior se planteó con el fin de clasificar la maquinaria por valor de inversión, luego, se realizó una simulación que ayudo a determinar cuál de las maquinarias escogidas requirió menor tiempo de ejecución en el proceso de revisión física del inventario [10] [11].

\section{Resultados}

Como se ha mencionado al inicio del presente artículo, el método utilizado actualmente por la compañía distribuidora no es el más seguro para el trabajo en alturas, incurre en altos costos y tiempos, por esto se pretende determinar la maquinaria y el método adecuado, teniendo en cuenta las siguientes etapas:

\section{- Diagnóstico del proceso actual de la revisión física del inventario.}

En esta primera etapa se realiza un estudio del trabajo con los tres métodos llevados a cabo por la compañía, esto con el fin de determinar el método óptimo para la revisión física del inventario.

Los tiempos son tomados desde que el operario sube a la canasta del montacargas hasta que termina la actividad.

Por cada método se realizaron tres pruebas, las cuales se clasificaron de la siguiente manera:

- P3_1, P3_2 y P3_3 es el número de pruebas realizadas con el método central.

- P1_1, P1_2 y P1_3 es el número de pruebas realizadas con el método central vertical.

- P2_1, P2_2 y P2_3 es el número de pruebas realizadas con el método zigzag.

Los resultados de este diagnóstico se pueden observar en tabla I.

En la primera columna se encuentran los métodos utilizados y el número de pruebas realizadas por cada método, en la segunda columna está el tiempo promedio por cada prueba de la lectura del UVA por cada nivel del rack en minutos y en la última columna se encuentra los mismos valores que en la segunda columna, pero en horas.

\begin{tabular}{|c|c|c|}
\hline Etiquetas de fila $\nabla$ & OPERARIO_(nivel/min) & OPERARIO_(nivel/h) \\
\hline$\Xi$ Central & 49,0 & 0,8 \\
\hline P3_1 & 49,4 & 0,8 \\
\hline P3_2 & 48,8 & 0,8 \\
\hline P3_3 & 48,9 & 0,8 \\
\hline$\Xi$ Central Vertical & 44,0 & 0,7 \\
\hline P1_1 & 45,5 & 0,8 \\
\hline P1_2 & 44,7 & 0,7 \\
\hline P1_3 & 41,8 & 0,7 \\
\hline$\ominus$ ZigZag & 30,7 & 0,5 \\
\hline P2_1 & 29,5 & 0,5 \\
\hline P2_2 & 32,2 & 0,5 \\
\hline P2_3 & 30,4 & 0,5 \\
\hline Total general & 41,2 & 0,7 \\
\hline
\end{tabular}

Fuente: Los autores, 2021. 
De acuerdo con los datos suministrados en la tabla I, se puede observar que el menor tiempo promedio le corresponde al método en Zigzag, con un tiempo de 30,7 min por nivel.

Para mejor comprensión de los datos obtenidos en la tabla I se pueden observar en la fig. 1.

Teniendo en cuenta la gráfica anterior se puede deducir que el método central equivale un $40 \%$ del tiempo promedio total de la operación, el método central vertical equivale un 35\% y el método Zigzag representa un $25 \%$, convirtiéndolo en el método con menos tiempo en la revisión física del inventario. Por lo tanto, el método zigzag es el método más eficiente.

\section{- Identificar la maquinaria para el proceso de re-} visión física del inventario

Al obtener el método viable para el proceso de la revisión física del inventario, el cual es el método zigzag, se continua con la búsqueda de máquinas alternas para el proceso de revisión física del inventario, donde se establecieron dos máquinas; el dron y el boogie, de la maquinaria nombrada anteriormente, se hacen dos estudios; uno de costos y otro de tiempos, con el fin de determinar el más apto para el proceso.

Para determinar los costos de la adquisición del boogie o dron, se tuvieron en cuenta: el costo de adquisición, el costo del alquiler, la hora del

\section{OPERARIO_(nivel/min) OPERARIO_(nivel/h)}

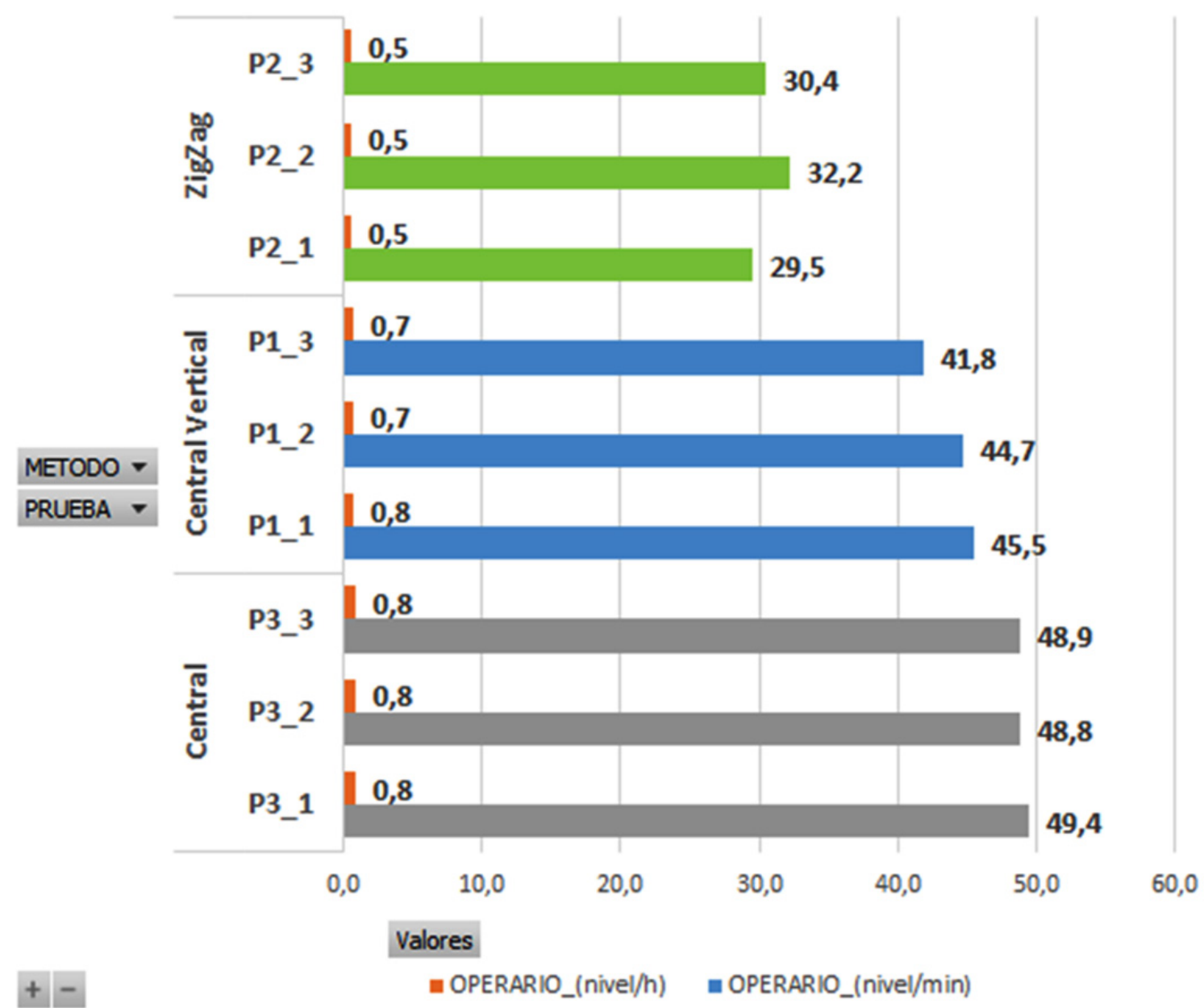

Fig. 1. Diagnóstico del proceso actual de la revisión física del inventario. Fuente: Los autores, 2021 
operario, el mantenimiento y la capacitación. Los costos son presentados en la tabla II.

Tabla Il. Costos de adquisición de maquinaria.

\begin{tabular}{|c|c|c|}
\hline \multicolumn{2}{|c|}{ Maquinaria } & Costo total \\
\hline \multirow{2}{*}{ Alquiler/Mes } & Dron & $\$ 566.667$ \\
\cline { 2 - 3 } & Boogie & $\$ 4.000 .000$ \\
\hline \multirow{2}{*}{ Compra } & Dron & $\$ 60.660 .600$ \\
\cline { 2 - 3 } & Boogie & $\$ 200.000 .000$ \\
\hline
\end{tabular}

Fuente: Los autores, 2021

Teniendo en cuenta los costos de adquisición al momento, del dron y del boogie, se puede deducir que el alquiler del dron es un $12,41 \%$ más económico en comparación con el boogie, en el caso de la compra, el dron es un $23,27 \%$ más económico que el boogie. Eso convierte al dron en la máquina más económica de adquirir o alquilar. Dando un mejor costo de oportunidad que el boogie.

Además del costo de adquisición, se tuvieron en cuenta los costos indirectos y directos de cada máquina, dando como resultado el que se muestra en la tabla III.

Tabla III. Costos directos e indirectos.

\begin{tabular}{|l|c|c|}
\hline \multicolumn{2}{|c|}{ Maquinaria } & Costo total \\
\hline \multirow{2}{*}{ Alquiler } & Dron & $\$ 11.128 .104$ \\
\cline { 2 - 3 } & Boogie & $\$ 16.750 .315$ \\
\hline Compra & Dron & $\$ 17.085 .938$ \\
\hline
\end{tabular}

Las proyecciones de estos costos se dieron para un periodo de 10 años que hace referencia a la vida útil de ambas maquinarias. En estos costos se debe tener en cuenta que:

- El mantenimiento del dron se debe realizar 4 veces al año, mientras que el boogie se realizaría una vez al año, donde el dron saldría 49,69\% más económico con respecto al boogie

- La capacitación se tiene en cuenta solo para la compra, en este caso el boogie es un $80,37 \%$ más económico que el dron.

De acuerdo los resultados en la tabla 2 y la tabla 3, se puede deducir que el dron es la opción más económica, ya sea para el alquiler o la compra, teniendo una diferencia del $12,41 \%$ y $23,27 \%$ respectivamente.

En este estudio también se pretendió determinar si es mejor la compra o alquiler de la maquinaria, para el caso del dron se puede deducir que es $49,56 \%$ más económica la compra que el alquiler del dron.

- Simulación de maquinaria

En la realización de la simulación se ambienta en una bodega con tres máquinas diferentes, que son: Dron, boogie y montacargas. Esta simulación costa de 3 racks, cada una con 5 niveles, 10 columnas y 160 módulos.

En la Fig. 2 se puede apreciar con más detalle los elementos utilizados para la simulación.

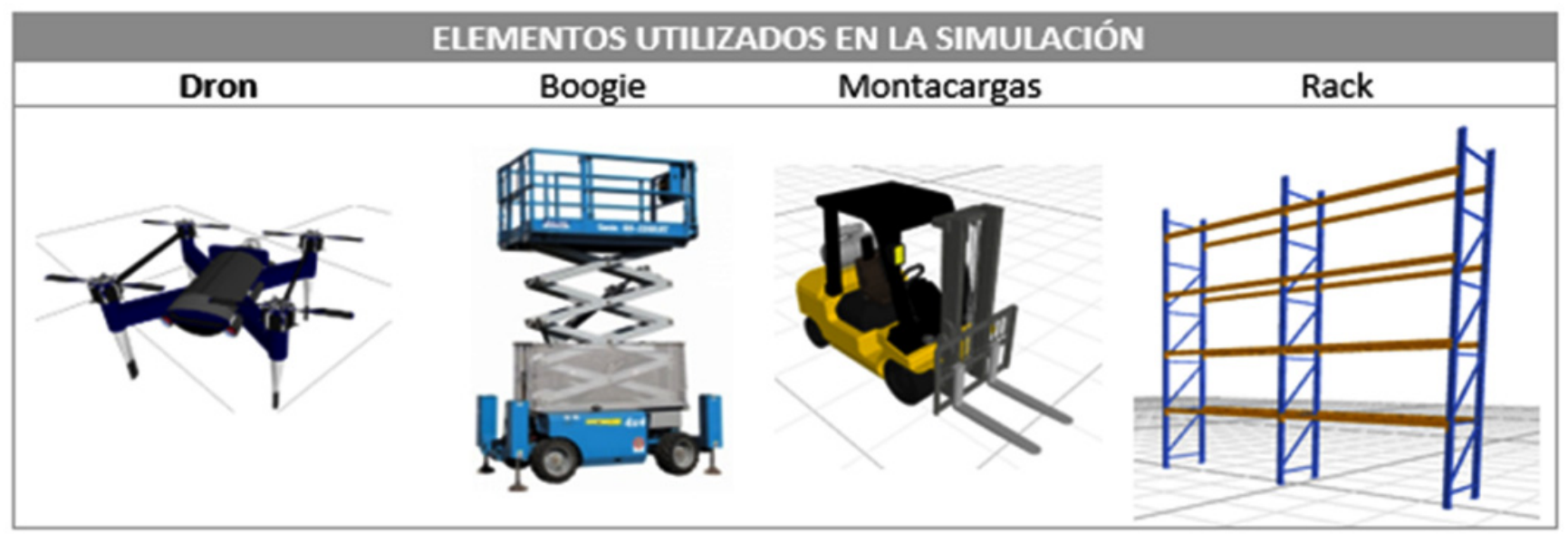

Fig. 2. Elementos de la simulación. Fuente: Los autores, 2021 
El tiempo utilizado para realizar la simulación con el montacargas, es el tiempo promedio por módulo (1,32 min), extraído del método Zigzag, el cual es el método más rápido y eficiente para la revisión física del inventario.

En el caso del dron, de acuerdo con diferentes consultas de los tiempos promedio en la industria y el desempeño de este en los procesos, se llegó a la conclusión que un dron se demora 0,65 minutos por módulo, siempre y cuando el dron tenga un lente con alto enfoque y una persona capacitada a la hora de manejarlo.

De igual manera sucede en los tiempos del boogie, su tiempo promedio en la industria es de 1,45 min por módulo. Este tiempo depende de la experiencia del operario a la hora de manejar el boogie y la eficiencia a la hora de tomar el UVA con el lector laser.

En la simulación se hace un plano 2D de la infraestructura, utilizando una entrada (sourse), un servidos (server) y una salida (sink). La simulación es realizada por 8 horas, el cual comprende una jornada laboral. La evidencia de la simulación se puede observar en la Fig. 3.

Luego de realizar la simulación, se obtienen los siguientes resultados referentes al rendimiento y tiempo en el sistema, los cuales se ven en la tabla IV.
Tabla IV. Rendimiento de simulación

\begin{tabular}{|c|c|c|c|c|}
\hline & $\begin{array}{c}\text { Tipo de } \\
\text { estadística }\end{array}$ & Valor & $\%$ & $\begin{array}{c}\text { Total } \\
\%\end{array}$ \\
\hline BOOGIE_1 & Total & 14,00 & $10 \%$ & \multirow{3}{*}{$29 \%$} \\
\hline BOOGIE_2 & Total & 12,00 & $8 \%$ & \\
\hline BOOGIE_3 & Total & 16,00 & $11 \%$ & \\
\hline DRON_1 & Total & 22,00 & $15 \%$ & \multirow{3}{*}{$48 \%$} \\
\hline DRON_2 & Total & 22,00 & $15 \%$ & \\
\hline DRON_3 & Total & 25,00 & $17 \%$ & \\
\hline MONTACARGA_1 & Total & 10,00 & $7 \%$ & \multirow{3}{*}{$22 \%$} \\
\hline MONTACARGA_2 & Total & 7,00 & $5 \%$ & \\
\hline MONTACARGA_3 & Total & 15,00 & $10 \%$ & \\
\hline \multicolumn{2}{|l|}{ TOTAL } & 143 & \multicolumn{2}{|c|}{$100 \%$} \\
\hline
\end{tabular}

Fuente: Los autores, 2021

El rendimiento da a conocer la productividad de cada una de las máquinas utilizadas en el sistema. De acuerdo con los datos, el 22\% del rendimiento en el sistema le corresponde al montacargas, el $29 \%$ al boogie y el $48 \%$ al dron. Siendo este el resultado final, es claro que la máquina más productiva en el sistema es el dron. Tabla V.

El tiempo de flujo, da a conocer el tiempo promedio de cada máquina en el sistema. Según los

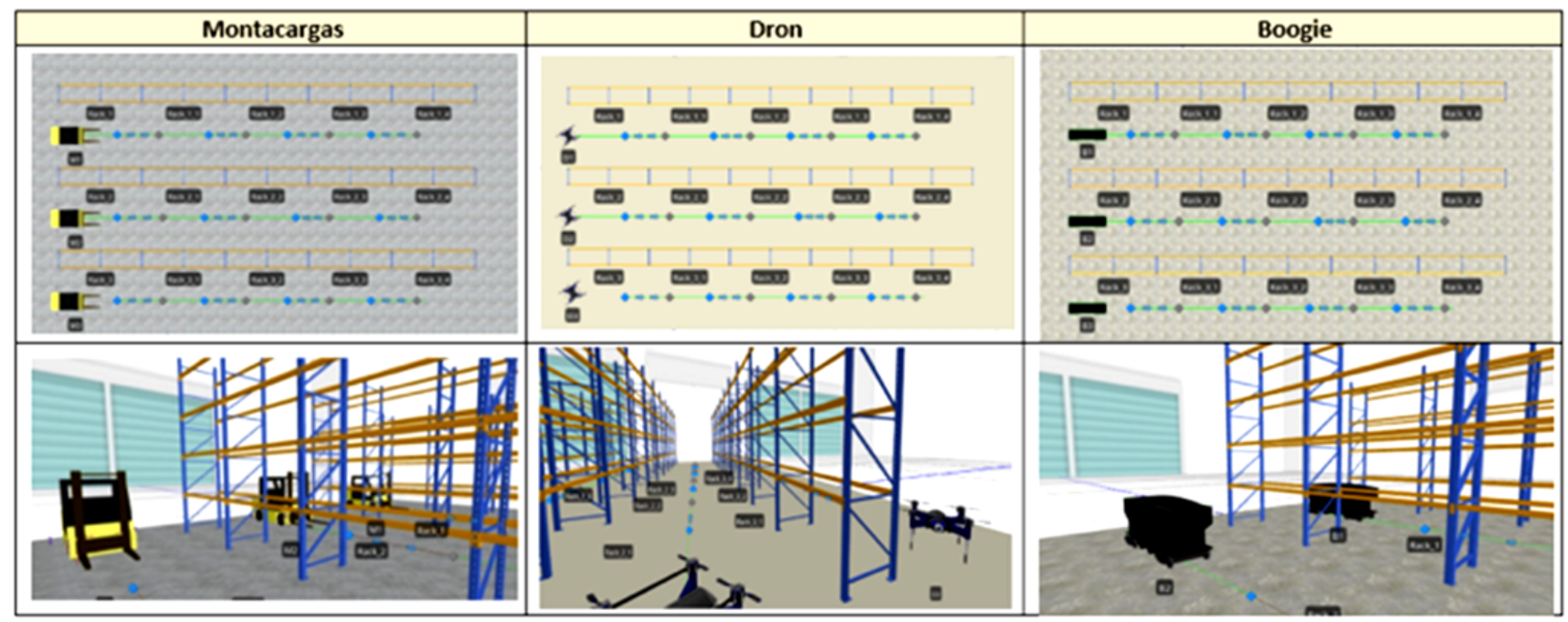

Fig. 3. Simulación por maquinaria. Fuente: Los autores, 2021 
Tabla V. Tiempo de flujo.

\begin{tabular}{|c|c|c|c|c|}
\hline Nombre del objeto & $\begin{array}{c}\text { Tipo de } \\
\text { estadística }\end{array}$ & Valor & $\%$ & $\begin{array}{c}\text { TOTAL } \\
\%\end{array}$ \\
\hline BOOGIE_1 & Promedio & 0,64 & $16 \%$ & \multirow{3}{*}{$40 \%$} \\
\hline BOOGIE_2 & Promedio & 0,48 & $12 \%$ & \\
\hline BOOGIE_3 & Promedio & 0,49 & $12 \%$ & \\
\hline DRON_1 & Promedio & 0,18 & $5 \%$ & \multirow{3}{*}{$14 \%$} \\
\hline DRON_2 & Promedio & 0,20 & $5 \%$ & \\
\hline DRON_3 & Promedio & 0,19 & $5 \%$ & \\
\hline MONTACARGA_1 & Promedio & 0,48 & $12 \%$ & \multirow{3}{*}{$46 \%$} \\
\hline MONTACARGA_2 & Promedio & 0,76 & $19 \%$ & \\
\hline MONTACARGA_3 & Promedio & 0,62 & $15 \%$ & \\
\hline \multicolumn{2}{|l|}{ TOTAL } & 4 & \multicolumn{2}{|c|}{$100 \%$} \\
\hline
\end{tabular}

Fuente: Los autores, 2021.

datos, el $46 \%$ del tiempo en el sistema le corresponde al montacargas, el $40 \%$ al boogie y el $14 \%$ al dron. El menor tiempo promedio en el sistema es el dron, esto lo convierte en la máquina más eficiente en el sistema, requiriendo menos tiempo para completar la tarea.

\section{CONCLUSIONES}

De acuerdo con el análisis de los resultados, la herramienta con más productividas y eficiencia en el sistema en cuestiones de tiempo, es el dron, opteniendo un $48 \%$ de productividad y un $14 \%$ de aprovechamiento en el tiempo.

La utilización de drones para la revisión física de inventarios disminuiría en un $100 \%$ el riesgo en trabajo en alturas.

El dron es la opción más viable, teniendo en cuenta los costos, el rendimiento, el tiempo y la seguridad del trabajador en el proceso.
El uso de la simulación para la comprobación de las hipótesis de solución se convierte en una herramienta necesaria y contundente para establecer la efectividad proyectada de las soluciones propuestas.

\section{REFERENCIAS}

[1] G. Kamawaty, "Introducción al estudio de trabajo" https://higieneyseguridadlaboralcvs.files. wordpress.com/2012/08/introduccic3b3n-al-estudio-del-trabajo-oit.pdf. 2012.

[2] COLPATRIA AXA, “Seguridad Industrial: Trabajo en alturas". https://www.arlcolpatria.co/ PortalUIColpatria/repositorio/AsesoriaVirtual/ a201512100454.pdf. 2015.

[3] ARLSURA, “Resolución 1409" https://www. arlsura.com/files/res1409_2012.pdf. 2012.

[4] A. Pérez. "Innovación tecnológica: tipos y características principales". https:// www.obsbusiness. school/blog/innovacion-tecnologica-tipos-ycarac teristicas-principales

[5] SIMIO, "inicio: simulación de procesos y operaciones" https://www.simiosimulacion.es/\#: :text $=$ SIMIO $\% 20$ es $\% 20$ un $\% 20$ software $\% 20$ de, $\log \%$ C3\% ADsticos \% 2C \% 20industriales \% 20y \% 20de $\% 20$ servicios

[6] M. López Trujillo and J. Correa Ospina, "Planeación estratégica de tecnologías informáticas y sistemas de información". Universidad de Caldas. Manizales-Colombia, 56p, 2007.

[7] J. Porto Pérez and M. Merino, “Definición de: Definición de racionalización" https:// definicion.de/ racionalizacion/

[8] "NTC ISO 31000 del 2018" https://sitios.ces. edu.co/Documentos/NTCISO31000_Gestion_ del_riesgo.pdf. 2018.

[9] Y.Duran, "Administración del inventario: Elemento clave para la optimización de las utilidades de la empresa" https:/ / www.redalyc.org/pdf/4655/ 465545892008.pdf. 2008.

[10] L. Krajewsky and L. Ritzman, “Administración de operaciones: estrategia y análisis," in Pearson educación, Boston, 154p, 2000.

[11] F. Mayer, "Estudio de tiempos y movimientos: Para manufactura gil," in Pearson educación, 168p, 2000. 
